\title{
EDIFICIO DE OFICINAS EN "TRES CANTOS” MADRID-ESPAÑA
}

\author{
(OFFICE BUILDING IN "TRES CANTOS”. MADRID-SPAIN)
}

Andrés Perea Ortega, arquitecto

Fecha de recepción: $16-\mathrm{V}-94$

$131-152$

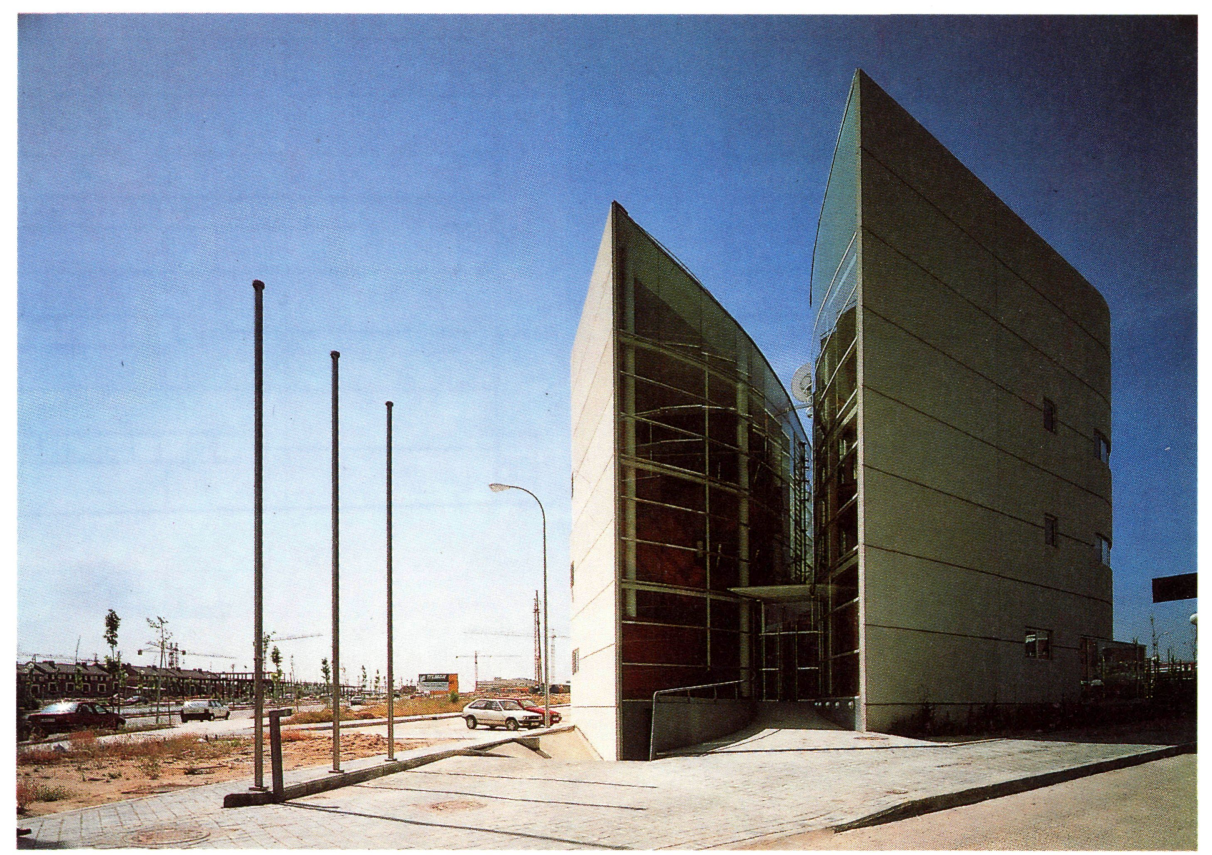

lista del edificio desde el acieso.

\section{RESUMEN}

En este artículo se detalla, sucintamente, el proyecto de un edificio de oficinas dividido, según el eje longitudinal, en dos piezas: tres plantas sobre rasante y tres plantas-sótano bajo rasante.

La idea del proyecto era crear un sistema ordenado del espacio desde los puntos de vista global, secuencial y dinámico.

\section{SUMMARY}

This article offers a brief description of an office building design. The building is divided along the longitudinal axis into two parts: three storeys above the groung level and three storeys below the ground level.

The idea of the design was to create an orderly system of space treatment from the global, sequential and dynamic points of view. 
El volumen definido por el planeamiento urbanístico se devide, según el eje longitudinal, en dos piezas; ambas soportan en las tres plantas, sobre rasante, un total de seis zonas con acceso independiente $y$, por tanto, comercializables por separado. Bajo rasante se proyectan tres plantas sótano que ocupan la totalidad del perímetro edificable.

\section{Accesos}

Los accesos desde el exterior se resuelven por el vértice norte del solar y del área edificable; tanto

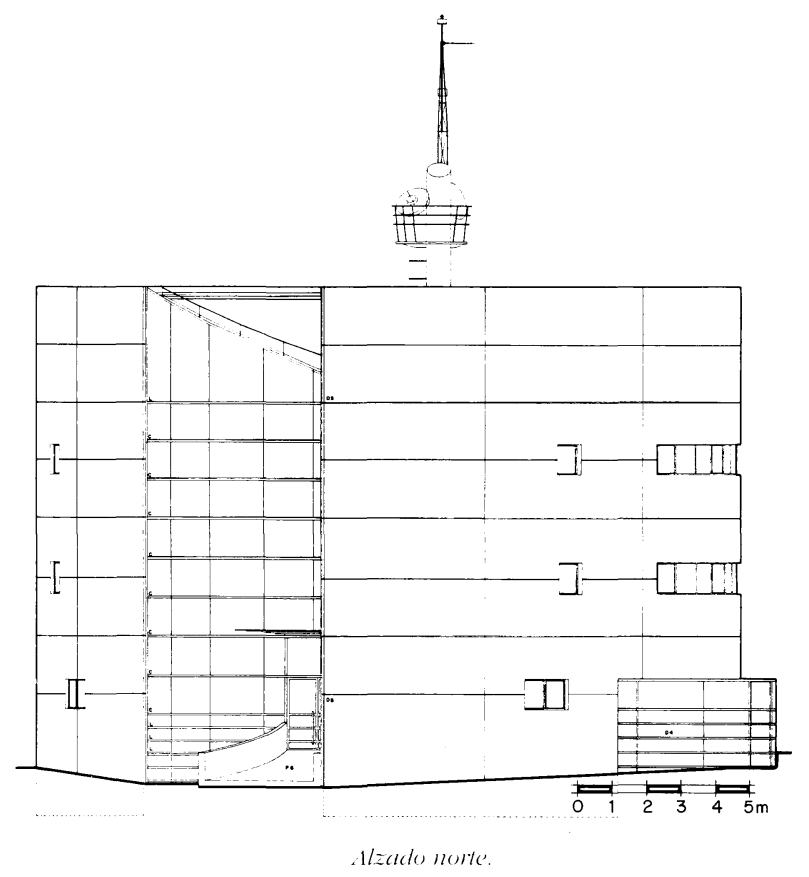

peatones como vehículos se introducen en el edificio por el espacio que separa los dos cuerpos de éste. Dos rampas conectan la cota de acceso con las correspondientes a la planta baja y al sótano. Los peatones acceden por la rampa descrita al núcleo de comunicaciones verticales y de control; los vehículos descienden al primer sótano y desde este nivel, por medio de rampas semicirculares, a los otros tres niveles. Un ascensor y escalera relacionan estas plantas bajo rasante con el acceso situado en planta baja. Los espacios libres de la parcela disponen de un acceso para vehículos, independiente y
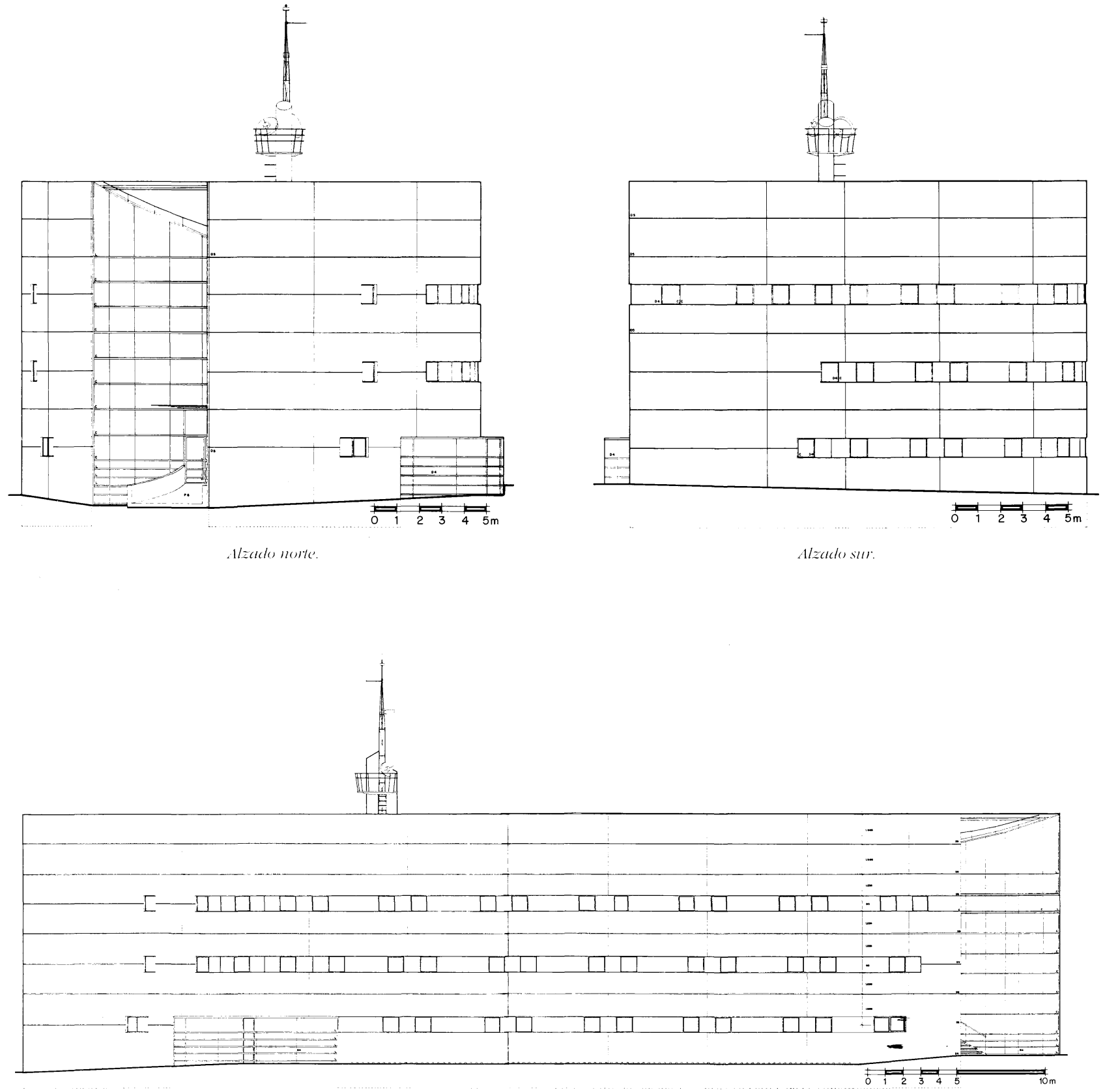
directo desde la vía pública, tanto para mantenimiento y acceso público como para el acceso del servicio de incendios a la fachada oeste.

\section{Plantas}

Las plantas sobre rasante se organizan de manera similar: el núcleo de circulación vertical situado en el extremo norte, los aseos de planta y los puestos de control de la misma se articulan sobre ese punto.
El espacio interior se plantea diáfano y con tres posibles sistemas de compartimentación: oficina paisaje total con subdivisiones mediante mobiliario, con circulación central y crujía exterior subdividida en despachos y la interior oficina paisaje o ambos lados compartimentados en forma de despachos y corredor de circulación central.

Las "medias plantas" en que ha resultado dividido el edificio, pueden relacionarse en un mismo nivel por puentes sobre el espacio abierto central.

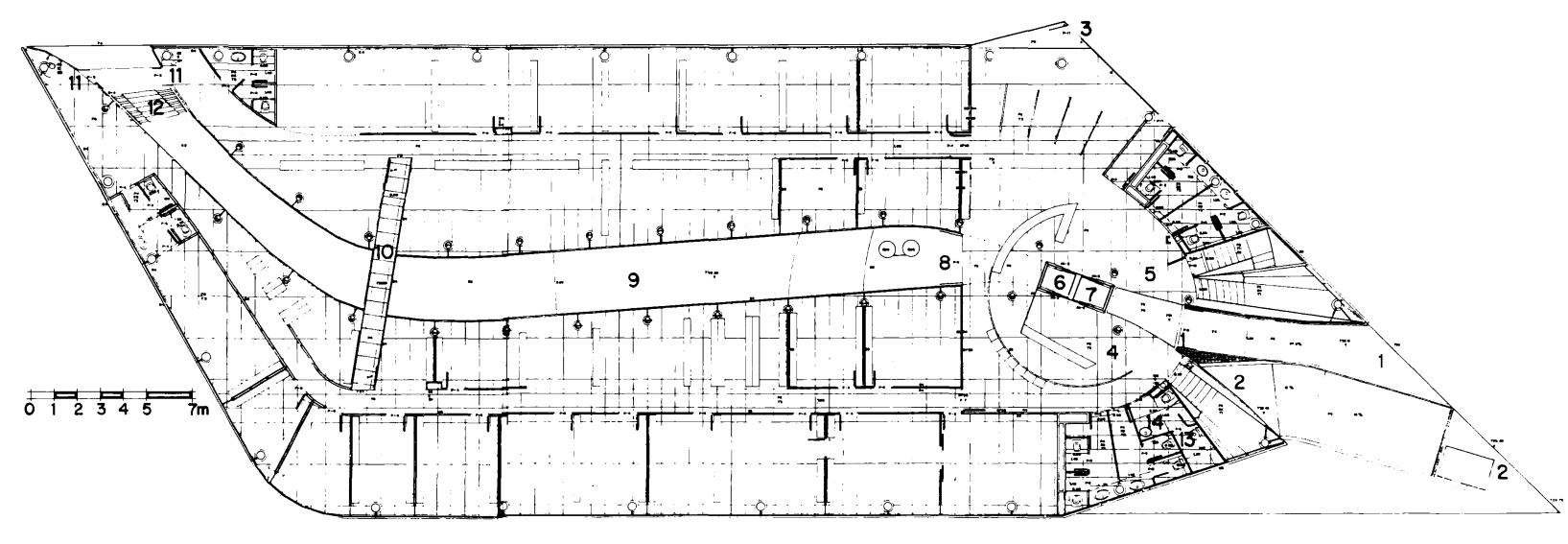

Planta baja

1: Acceso general peatones. 2: Rampa acceso vehículos. 3: Acceso independiente a oficinas de Tres Cantos, S.A. 4: Vestíbulo general del edificio. 5: Vestíbulo de las oficinas de Tres Cantos, S.A. 6: Ascensor de conexión planta baja a sótanos. 7: Ascensor de conexión planta baja a plantas superiores. 8: Salida a patio interior. 9: Patio interior-estanque. 10: Pasarela. 11: Salidas de emergencia. 12: Escaleras de emergencia. 13: Cuarto de limpieza. 14: Aseo minusválidos.

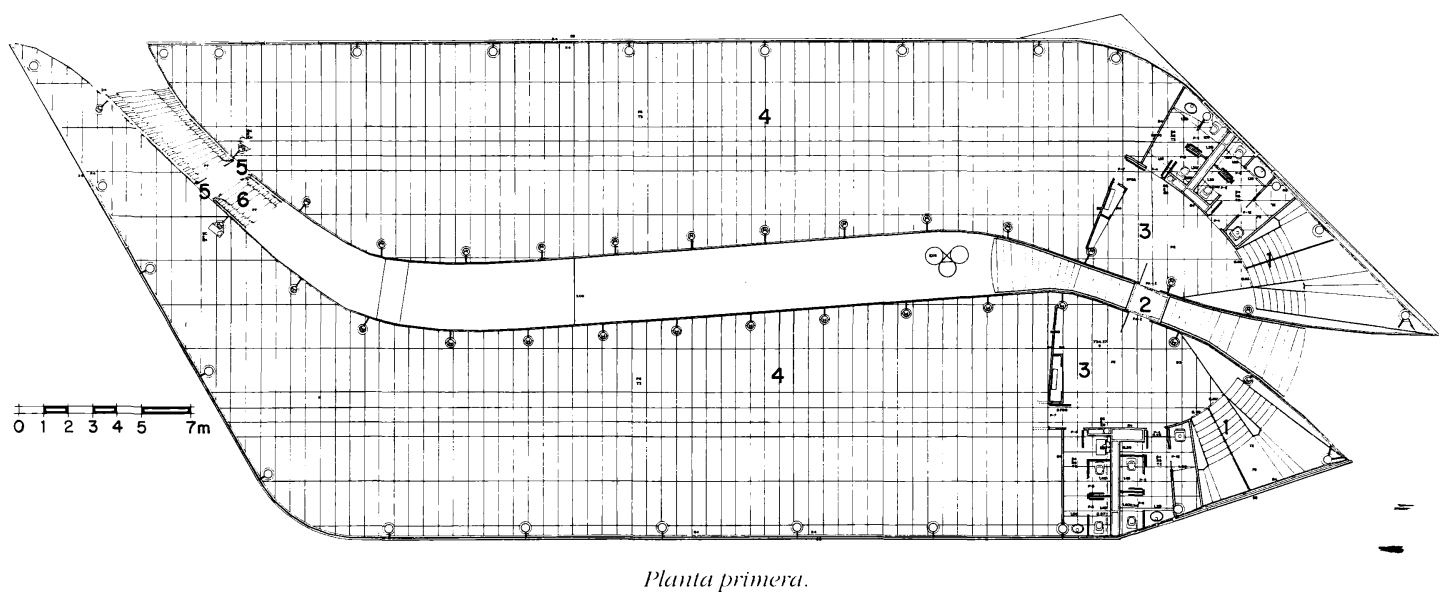

1: Escaleras conexión con planta baja. 2: Ascensor hidráulico conexión con planta garaje. 3: Vestíbulo de control e información. 4: Zona de oficinas. 5: Salidas de emergencia. 6: Escalera de emergencia. 


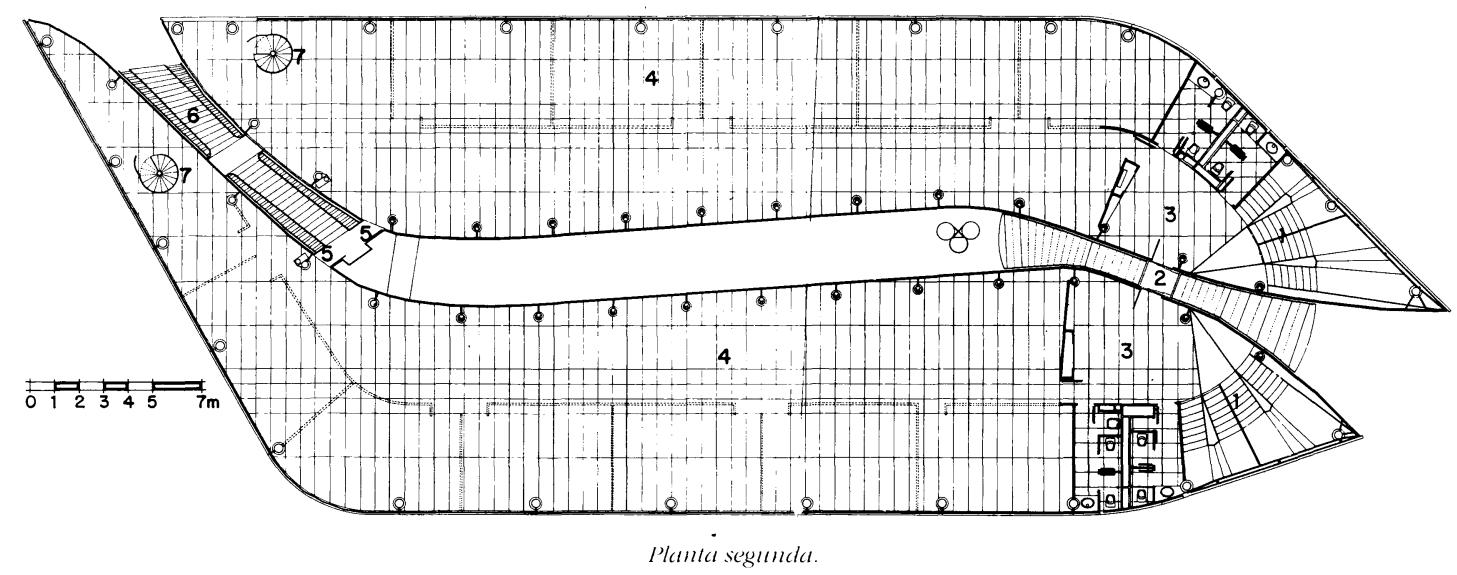

1: Escaleras conexión con planta baja. 2: Ascensor hidráulico conexión con planta garaje. 3: Vestíbulo de control e información. 4: Zona de oficinas. 5: Salidas de emergencia. 6: Escalera de emergencia. 7: Escaleras de acceso a cámara bajo cubierta.

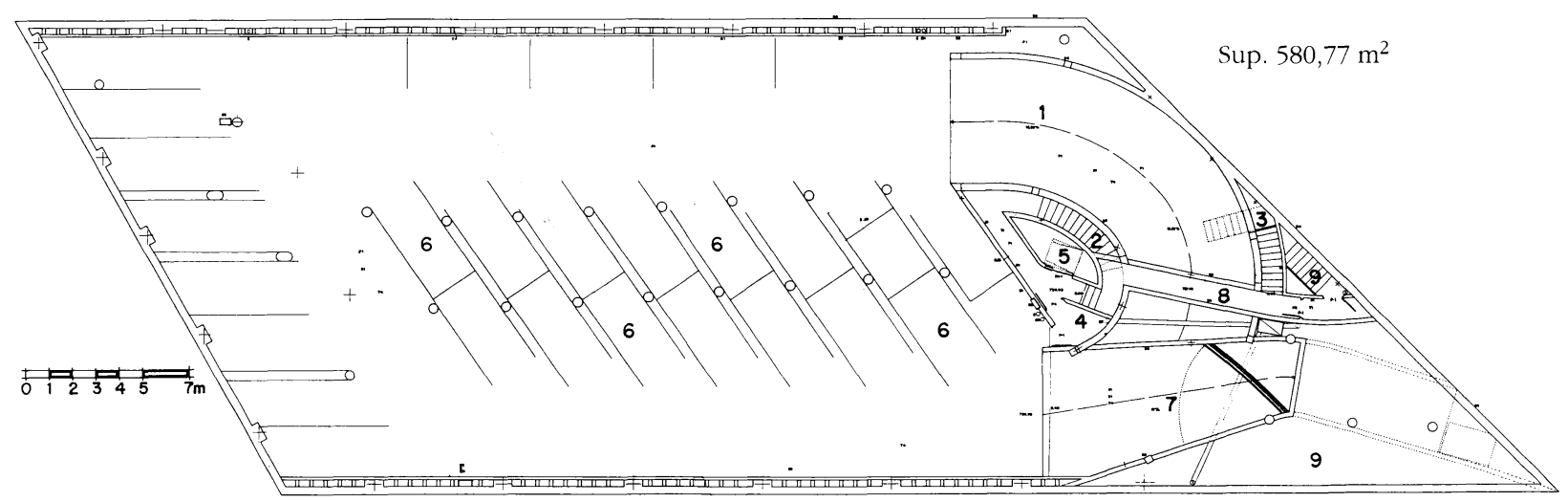

Sótano primero.

1: Rampa de acceso a garaje. 2: Escalera de conexión de garaje con planta baja. 3: Escalera de conexión de salas de máquinas con planta baja. 4: Vestíbulo. 5: Ascensor hidráulico de sótanos a planta baja. 6: Garaje-aparcamiento. 7: Rampa salida al exterior. 8: Salida al portal. 9: Vacío sobre sótano segundo.

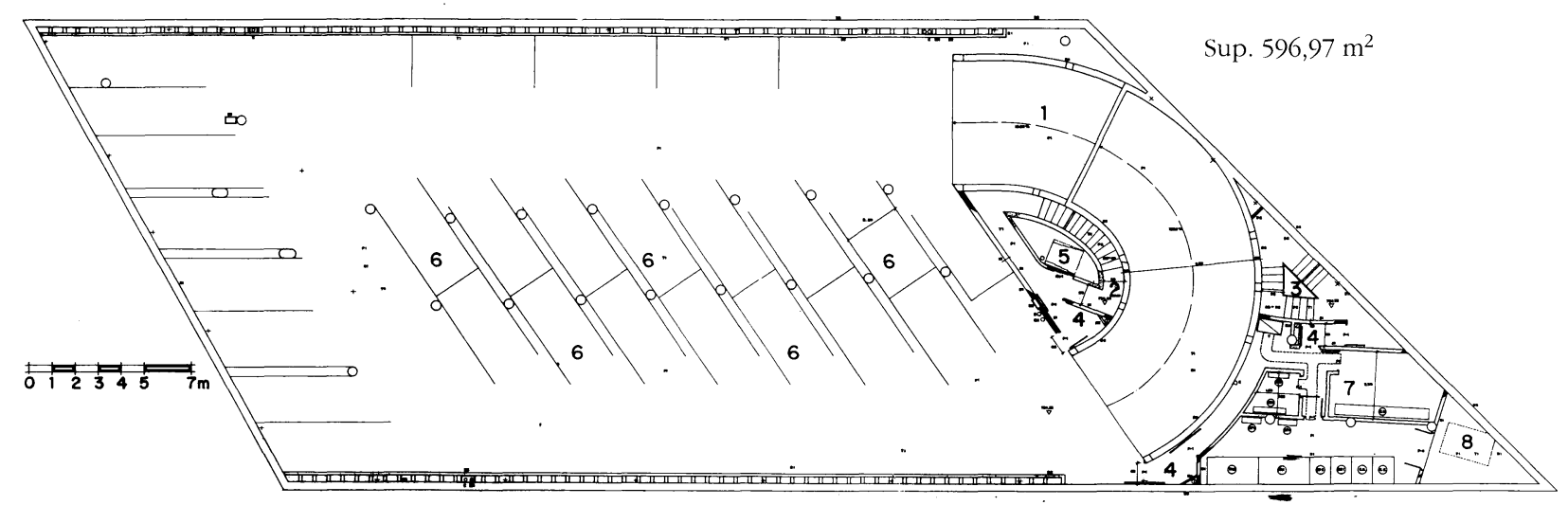

Sótano segundo.

1: Rampa de acceso a garaje. 2: Escalera conexión garaje a planta baja. 3: Escalera conexión salas de máquinas a planta bajo. 4: Vestíbulos. 5: Ascensor hidráulico de sótanos a planta baja. 6: Garaje-aparcamiento. 7: Centro de transformación. 8: Posible patio entrada de equipos. 


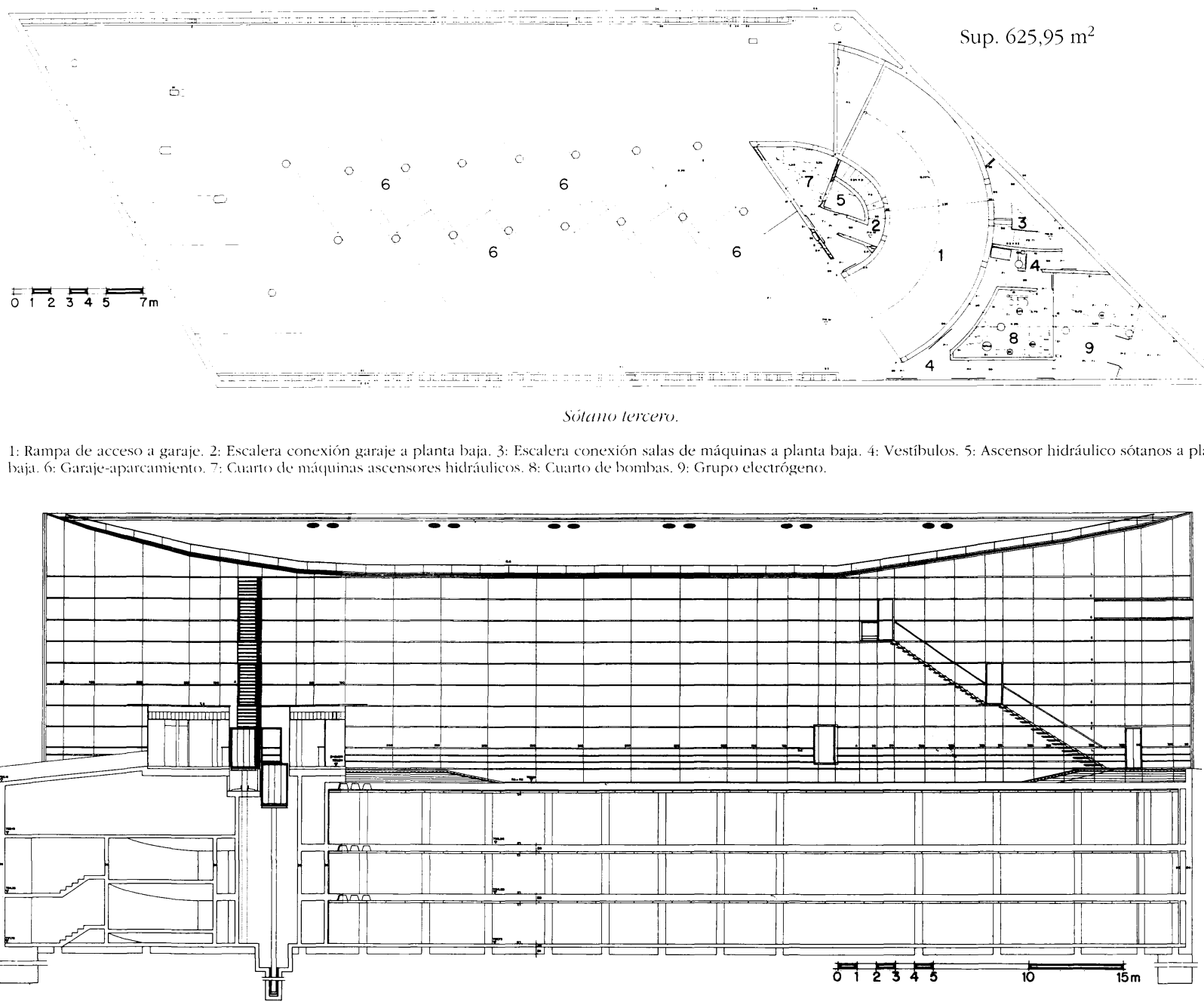

Sección longitudinal desarrollada $A A$

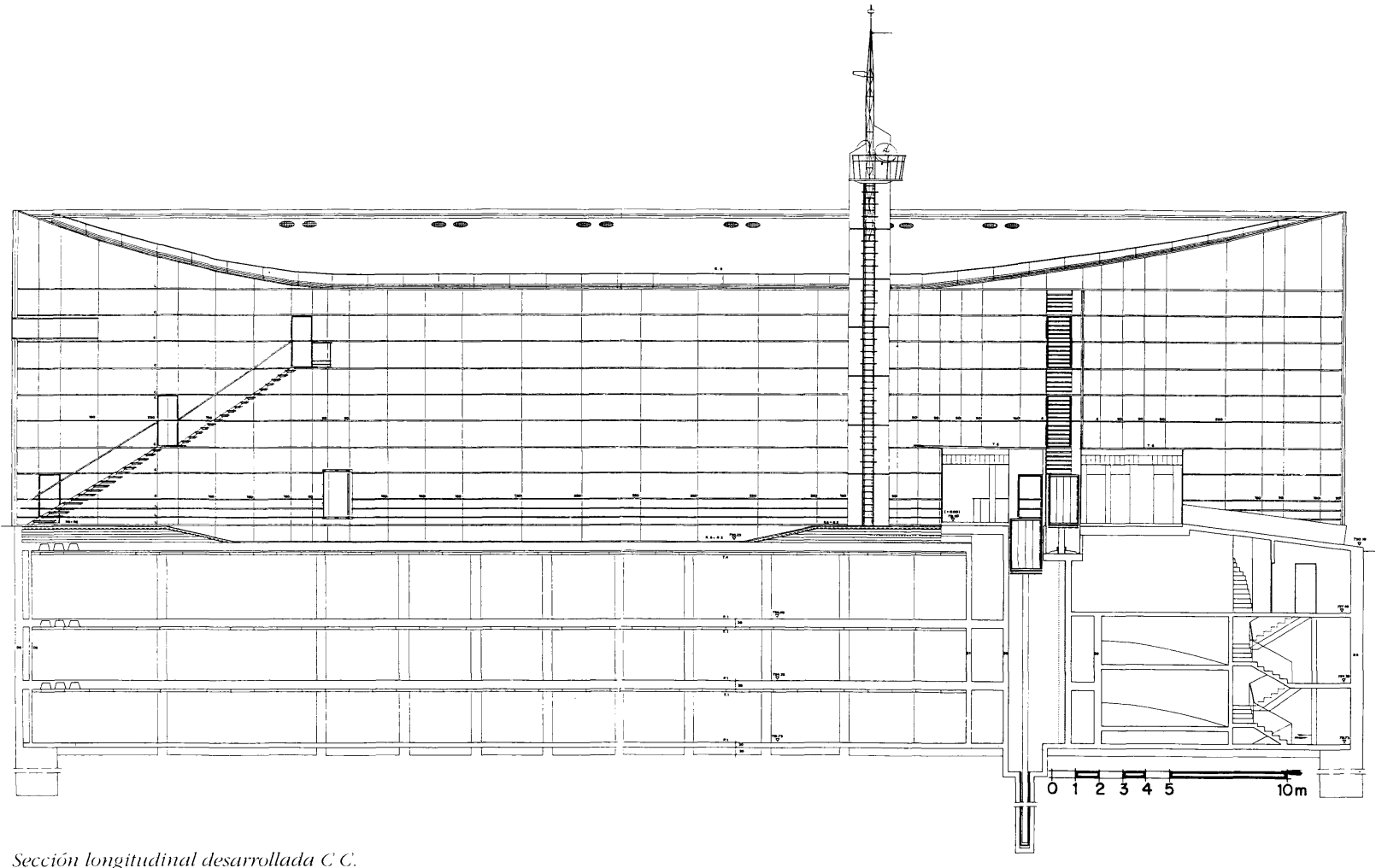

(c) Consejo Superior de Investigaciones Científicas 


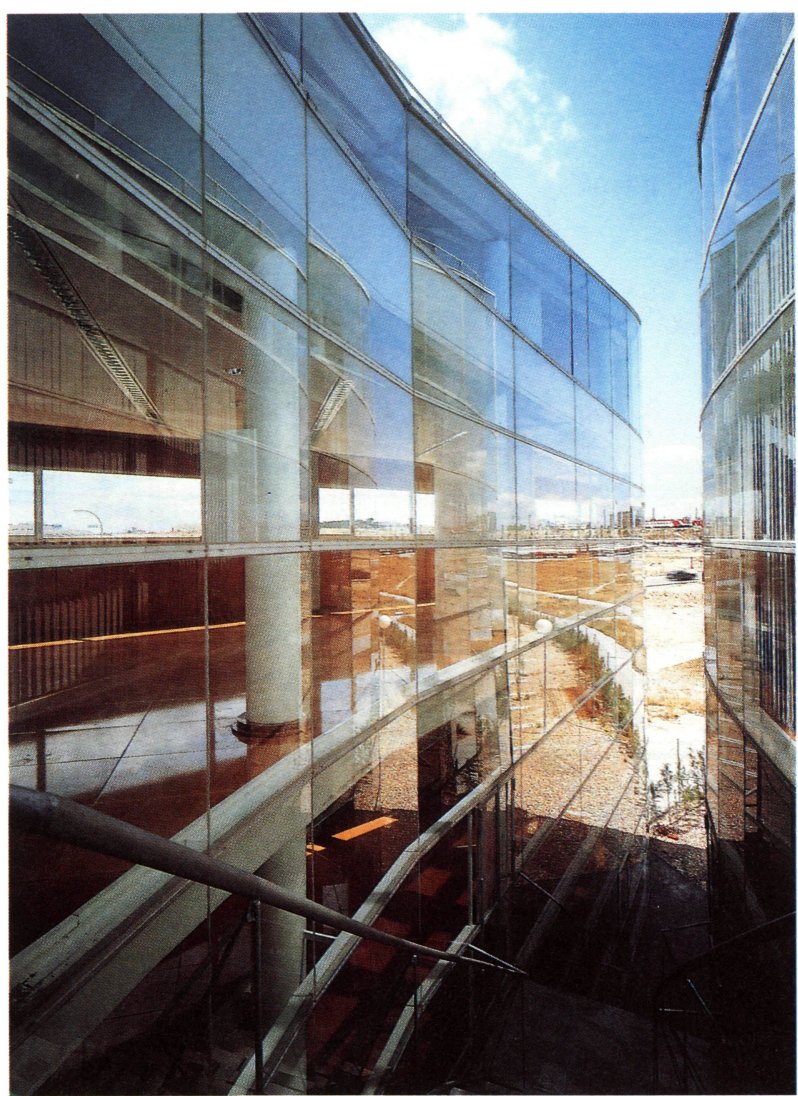

El patio interior.

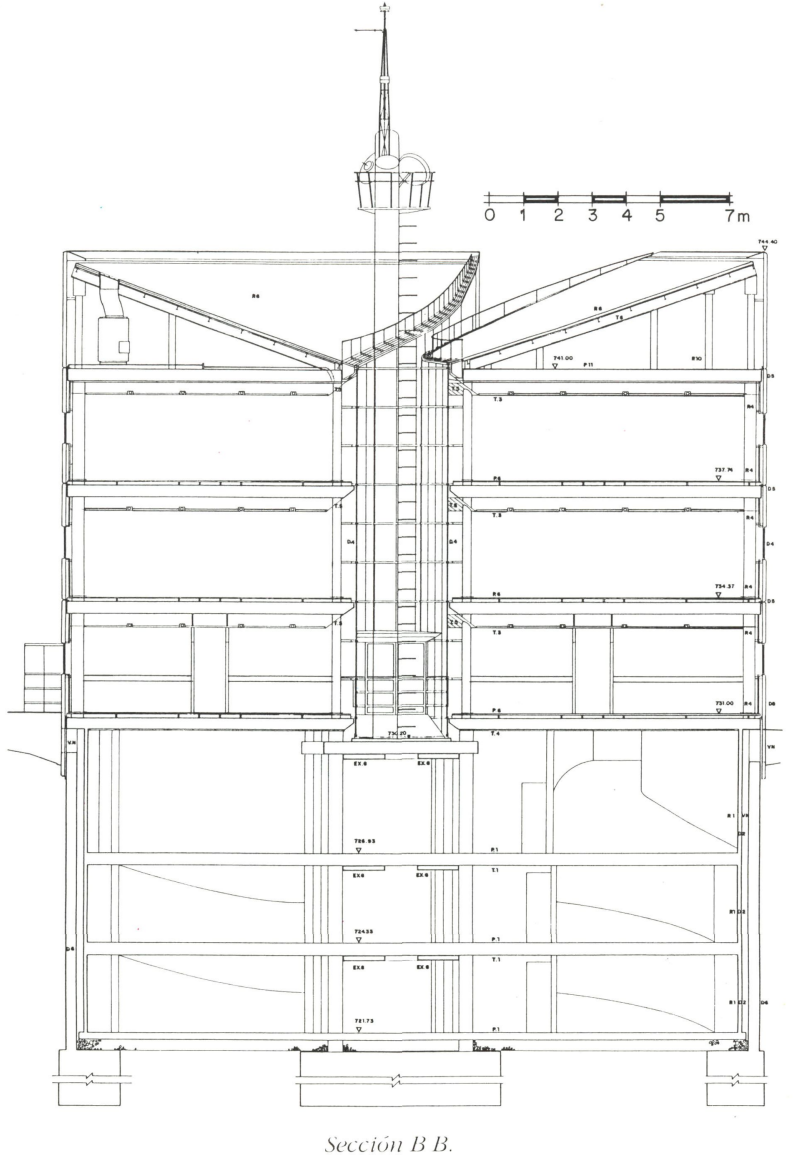

\section{Justificación del proyecto}

Desde el punto de vista formal, este proyecto se justifica en la necesidad de motivar un volumen edificable injustificado aun desde los supuestos de un urbanismo de planta y gran escala. El Plan Parcial supone que un volumen de tres plantas construye, junto a volúmenes similares, "fachadas" del polígono. Sin embargo, lo cierto es que las escasas dimensiones y la heterogeneidad morfológica esperable de la promoción por distintas iniciativas privadas producirá inevitablemente una simple suma "alineada" de edificios aislados en el territorio.

Este proyecto asume esta realidad final y propone un edificio que formalmente plantea sus propias reglas de expresión. El carácter no habitacional del edificio es el primer mensaje a emitir. La orientación de las fachadas longitudinales oeste-este implica la defensa del excesivo asoleo sobre el espacio interno y, por tanto, la estricta apertura de mínimos huecos en estas superficies exteriores; el resultado es el segundo mensaje sobre el carácter centrípeto del espacio contenido. Espacialmente, el proyecto se justifica como un sistema jerarquizado de tratamiento del espacio, desde las categorías espaciales de carácter global y secuencial o dinámica.

El espacio central es un desfiladero de cristal, gran grieta perfilada por suaves superficies de vidrio rutilante vacío formal que cohesiona el entendimiento parcial de los elementos formales descritos.

En este sentido y pretendiendo hacer inequívoco el carácter centrípeto del espacio definido por las superficies exteriores, en el diseño de éstas se obvian los encuentros en ángulo de las fachadas y son tratadas como planos verticales que se doblan y curvan según directrices de sector de círculo, como 


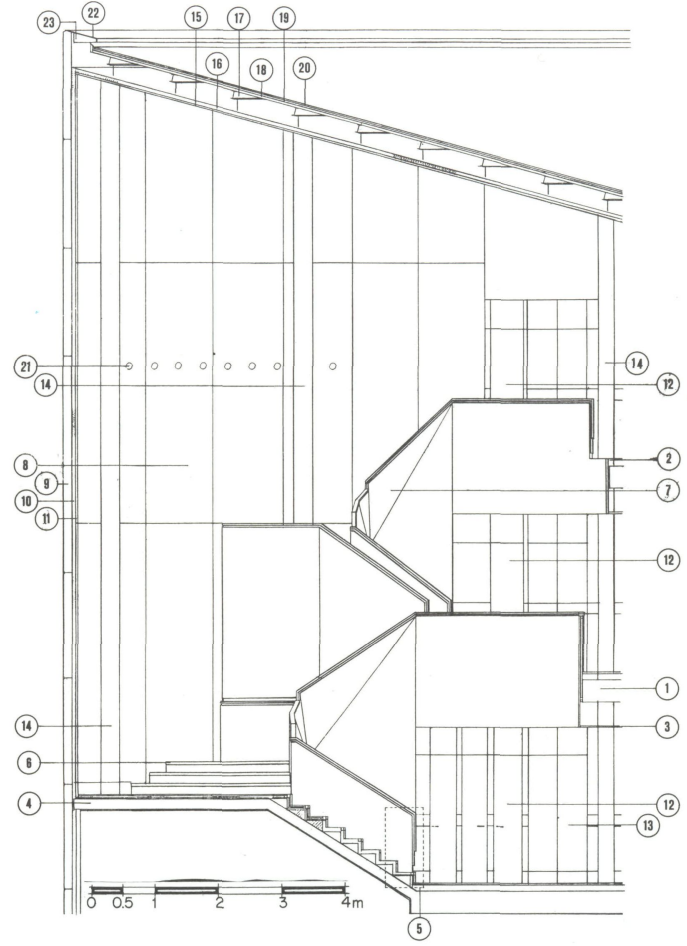

Alzado escalera

1: Forjado. 2: Suelo flotante. 3: Falso techo. 4: Losa de escalera. 5: Ver plano D-28. 6. Mamperlán de haya 7. Planos cubrehuecos en pyrelite 8. Tablero de pyrelite. 9. Panel de hormigón prefabricado. 10: Aislante $(6 \mathrm{~cm})$. 11: Tablero de pyrelite. 12. Puerta de aseo. 13. Frente patinillo desmontable 14: Pilar con funda de chapa 12: Puerta de aseo. 13: Frente patinillo desmontable. 14: Pilar con funda de chapa 15: Techo de pladue. 16: Aislante. 17: TPN 120. 18: Chapa nervada. 19: Capa de hormi22. Chapa $(3 \mathrm{~mm})$ 23: Cartabón rigidizador. si el espacio interno y su límite exterior hubieran encontrado su estado de equilibrio.

Efectivamente, el proyecto plantea un programa espacial global, riguroso y unívoco: el espacio exterior y el interior están escindidos radicalmente en las fachadas este y oeste y, por el contrario, se permeabilizan todo lo posible en el eje norte-sur. En esta dirección el espacio exterior hiere el edificio según espina dorsal y el espacio interno pretende capturar esa franja del espacio abierto, apresando así una atmósfera abierta peculiar. El perímetro de este espacio interno, de traza irregular y facetado de vidrio, se espera que sea rutilante y variable con el estado exterior del día y el ciclo estacional. Así, las áreas internas de trabajo están físicamente escindidas por esta fractura, pero virtualmente unidas por la visión inmediata entre ambas partes.

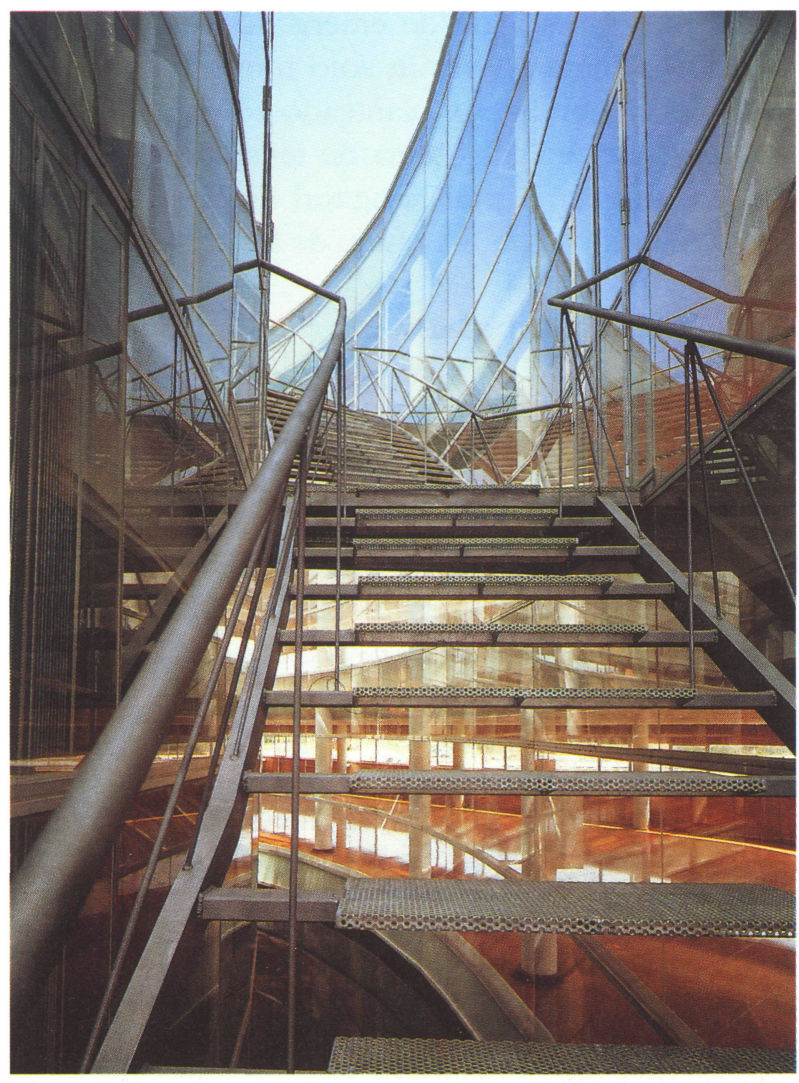

La escalera de emergencia en el lado opuesto al acceso.

\section{Elementos de circulación}

Los elementos de circulación vertical están planteados con un decidido protagonismo en la percepción dinámica del espacio. Los ascensores, simples cabinas de vidrio proyectadas al aire libre y accionadas por un émbolo central, se deslizan entre las paredes del espacio central, en tanto que en situación de reposo este ámbito queda libre de obstáculos.

Las cabinas, por otra parte, son un auténtico vehículo ascensorial desde el que percibir la dialéctica interior-exterior que argumenta todo el programa espacial.

Las escaleras, organizadas funcionalmente en dos piezas independientes constituyen una única entidad espacial que, a partir del eje de acceso, se articulan en quiebros de ida y vuelta, desplazados de forma que este recorrido es un desplazamiento espacial de gran entidad. 
Finalmente, la escalera de emergencia del extremo sur es un elemento de un solo tramo de material extremadamente ligero, planteado como resolución simple y directa del sistema de tensiones perceptivas que origina la gran longitud de la planta y la localización de accesos en uno de los extremos.

\section{Espacio libre y arbolado}

El tratamiento del espacio libre de la parcela es muy elemental, y se cree que eficaz; se organiza en franjas, sensiblemente orientadas en dirección norte-sur limitadas por un sistema de canales y fuentes, que proporcionan el sistema básico sobre el que proyectar arbolado, fuentes, arbustos, macizos de flores, etcétera, y finalmente un posible espacio de esparcimiento deportivo.

La utilización del arbolado, como un denso y alto muro vegetal de hoja caduca, cumple la función de filtro al soleamiento estival de poniente y permeabiliza el invernal más deseable en el interior.

\section{Ficha técnica}

\begin{tabular}{|c|c|c|c|}
\hline Situación: & $\begin{array}{l}\text { Parcelas } 11 \text { y } 12 \text { de la Unidad de Actuación UTA-4, en Tres Cantos. } \\
\text { Madrid. }\end{array}$ & Colaboradores: & $\begin{array}{l}\text { Capitolino González Rodrigue\%. Luis González Mariscal } \\
\text { y Miguel Hernández Lope\%, arquitectos. }\end{array}$ \\
\hline \multirow[t]{2}{*}{ Arquitecto: } & Andrés Perea Ortega. & Arquitecto Técnico: & Julio Hernanz Cabilla. \\
\hline & & Empresa Constructora: & GINES NAVARRO. S.A. \\
\hline
\end{tabular}

\section{Publicaciones del Instituto Eduardo Torroja-CSIC}

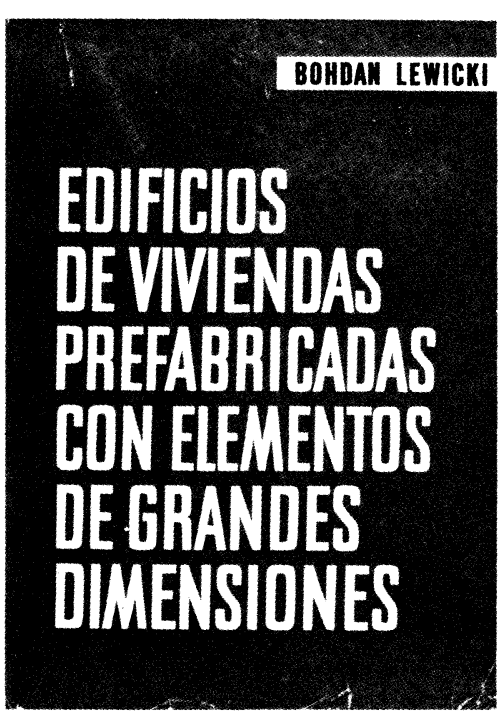

\section{Bohdan Lewicki}

Este libro trata de los problemas relativos a la construcción de los edificios de viviendas o publicos realizados con elementos prefabricados de grandes dimensiones. Se han estudiado los problemas de arriostramiento, asi como los que plantea la resistencia de los elementos y de la estructura; se han examinado las cuestiones de orden higrotermico, acústico y de resistencia al fuego; tambièn se ha profundizado en el estudio de la estanquidad de los muros exteriores y de las juntas.

La obra incluye numerosas ilustraciones que dan detalles de diversas soluciones, asi como ejemplos de cálculo, tablas de valores numéricos, diagramas y ábacos.

Un volumen encuadernado en tela, de $24 \times 17 \mathrm{~cm}$, compuesto de 616 págs.
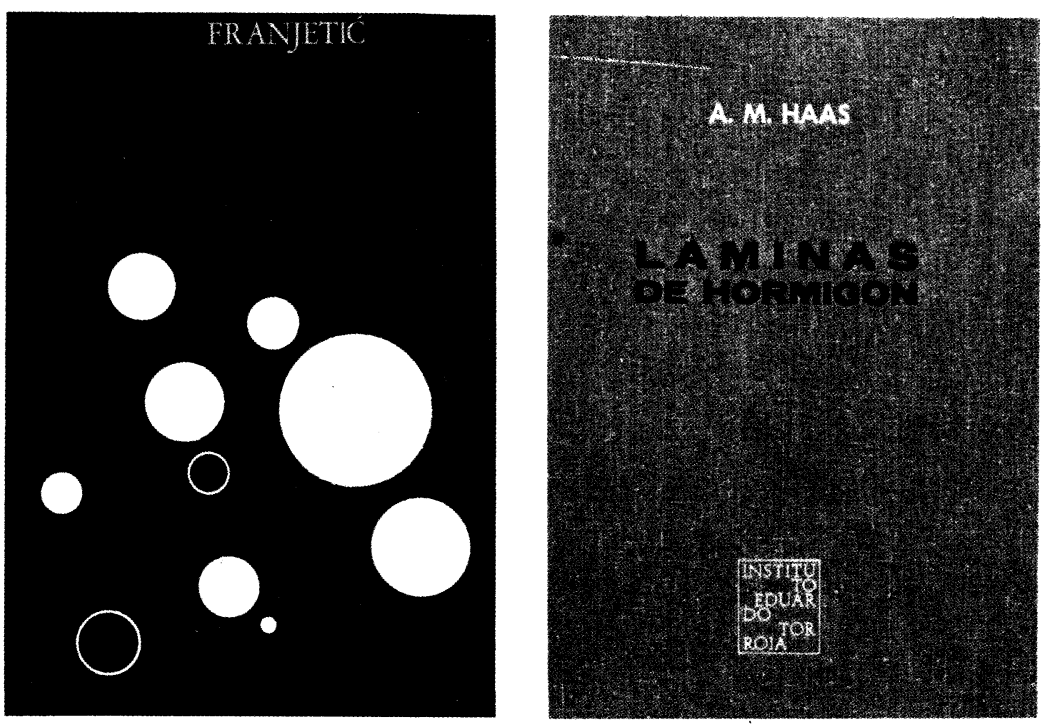

\section{Zorislav Franjetić}

En la obra de Franjetić se expone de una forma minuciosa, ordenada y sistemática, todo un cuerpo de doctrina que reúne el conocimiento actual sobre el endurecimiento rápido del hormigón. Parte el autor de los principios básicos y llega a las últimas conprincipios básicos y llega a las últimas con-
secuencias y realidades tecnicas y econo micas.

Es una obra de consulta, tanto para el investigador sobre la materia, como para el proyectista y el realizador y montador de plantas $e$ instalaciones y equipos de curado y endurecimiento rápido.

Un volumen encuadernado en cartóné, de $17 \times 24,5 \mathrm{~cm}$, compuesto de 385 págs. 110 figuras y 10 tablas.

\section{A. M. Haas}

Al escribir este libro el autor intento poner a disposición de los estudiantes y de los ingenieros unos conocimientos prácticos. adecuados para servir de guia en el diseño y construcción de láminas delgadas de hormigón.

El autor está convencido de que el éxito en el diseño de una lámina exige, por parte del proyectista, un examen de las tres fases por las que pasa la materialización de la lámina: el diseño, el análisis estructural y la construcción de la estructura.

Un volumen encuadernado en tela, de $17 \times 24,5 \mathrm{~cm}$, compuesto de 420 págs., 141 figuras, 22 fotografias y 6 tablas. 